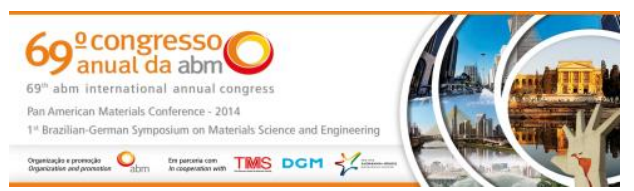

Tema: Materiais com Efeito de Memória de Forma

\title{
INFLUÊNCIA DA ECAE NAS PROPRIEDADES DE LIGAS NiTi COM EFEITO DE MEMÓRIA DE FORMA*
}

\author{
Niédson José da Silva \\ Carlos Augusto do Nascimento Oliveira ${ }^{2}$ \\ Cezar Henrique Gonzalez ${ }^{3}$ \\ Carlos José de Araújo 4 \\ Oscar Olímpio de Araújo Filho ${ }^{5}$ \\ Leonardo Mendonça Carneiro da Silva ${ }^{6}$
}

\section{Resumo}

Ligas com memória de forma (LMF) são materiais ativos que apresentam como característica a resposta a impulsos externos, recuperando deformações aparentemente plásticas. Esses materiais têm forte potencial aplicativo como atuadores termomecânicos em diversos setores industriais, sendo de interesse o aperfeiçoamento de suas propriedades termomecânicas. A conformação mecânica do ECAE (Equal Channel Angular Extrusion) resulta em melhorias quanto às propriedades mecânicas destas ligas. Esta melhoria está relacionada à redução de tamanho de grãos devido ao cisalhamento interno que estes sofrem ao passar pelos canais interceptantes, apesar de não ocorrer nenhuma alteração na área da seção transversal do corpo de prova. Neste trabalho, realizou-se a fabricação de ligas com memória de forma da família NiTi e da matriz para a extrusão em canal angular. A conformação mecânica foi realizada numa máquina de ensaios universal. O resultado desse estudo foi a observação da melhoria das propriedades mecânicas e alteração das temperaturas de transformação, as quais são essenciais para a utilização do efeito de memória de forma. Essa análise se torna relevante quando da aplicação desse tipo de material em situações em que se requer elevada resistência mecânica aliada a eficácia dos fenômenos de memória de forma.

Palavras-chave: Ligas NiTi; ECAE; Temperaturas de transformação; Conformação mecânica.

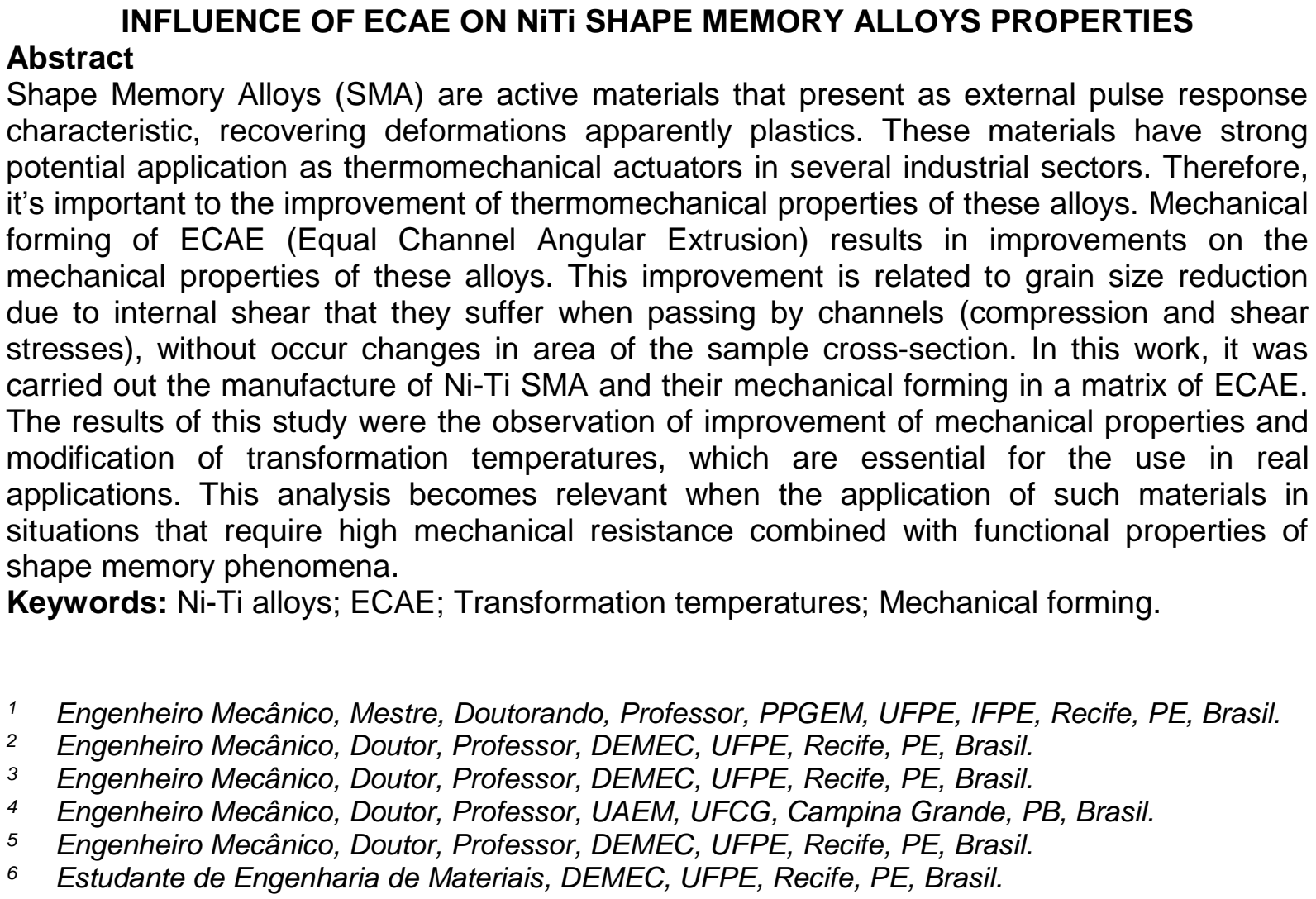
characteristic, recovering deformations apparently plastics. These materials have strong potential application as thermomechanical actuators in several industrial sectors. Therefore, it's important to the improvement of thermomechanical properties of these alloys. Mechanical forming of ECAE (Equal Channel Angular Extrusion) results in improvements on the mechanical properties of these alloys. This improvement is related to grain size reduction due to internal shear that they suffer when passing by channels (compression and shear stresses), without occur changes in area of the sample cross-section. In this work, it was carried out the manufacture of Ni-Ti SMA and their mechanical forming in a matrix of ECAE. The results of this study were the observation of improvement of mechanical properties and modification of transformation temperatures, which are essential for the use in real applications. This analysis becomes relevant when the application of such materials in situations that require high mechanical resistance combined with functional properties of shape memory phenomena.

Keywords: Ni-Ti alloys; ECAE; Transformation temperatures; Mechanical forming.

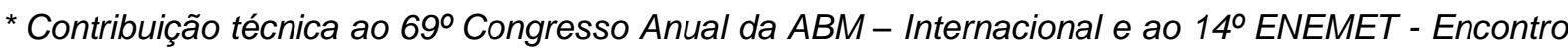
Nacional de Estudantes de Engenharia Metalúrgica, de Materiais e de Minas, 21 a 25 de julho de 2014, São Paulo, SP, Brasil. 


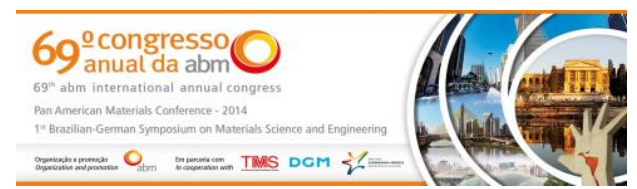

\section{INTRODUÇÃO}

As Ligas com Memória de Forma (LMF) são materiais ativos que apresentam a capacidade de recuperar deformações aparentemente plásticas através de alterações de temperatura elou de esforço externo. Este fenômeno, designado por Efeito de Memória de Forma (EMF), está intimamente associado a uma transformação de fase do tipo martensítica, cristalograficamente reversível [1]. A fabricação das ligas com memória de forma da família $\mathrm{Ni}-\mathrm{Ti}$ é especialmente importante devido ao fato de pequenas variações na composição química podem alterar as temperaturas de transformação martensítica em cerca de até $100^{\circ} \mathrm{C}$ [2]. Portanto, o processo de fabricação por plasma é utilizado de forma eficiente pelo fato de provocar perda insignificante de material por oxidação durante a fusão, o que pode validar o método estudado por Araújo et al. [3]. As ligas da família Ni-Ti são amplamente utilizadas devido à sua extensiva gama de possibilidades de aplicações tecnológicas, que se estendem da área biomédica à aeroespacial e petróleo [4-9].

As LMF são consideradas atuadores elétricos quando o EMF é ativado através da aplicação de pulsos de corrente elétrica (efeito Joule). Em contrapartida, se o EMF é ativado diretamente por meio da variação controlada da temperatura, consideram-se como sendo atuadores térmicos. Essas ligas quando submetidas a processos de conformação mecânica, ou seja, submetidas a deformações por meios mecânicos, demonstram tendência ao aumento de sua resistência mecânica devido tanto ao encruamento quanto a redução do tamanho de grãos. Esta alteração das propriedades mecânicas provoca também uma mudança nas temperaturas de transformação, característica essencial para utilização desses materiais em aplicações tecnológicas.

A ECAE (Equal Channel Angular Extrusion) ou ECAP (Equal Channel Angular Pressing) é um tipo de conformação recentemente desenvolvido por Segal [10], que consiste em promover a passagem de material de uma extremidade à outra de dois canais sequenciais que se interceptam formando entre si ângulos entre $90^{\circ}$ e $120^{\circ}$. Nesse tipo de processo uma deformação plástica severa provoca a aplicação de uma grande quantidade de tensão crítica de cisalhamento sem modificação da seção transversal do material [11]. Alguns estudos demonstram que os fenômenos de deformação que acontecem durante o processo de ECAE são independentes do tamanho da amostra, o que sugere aplicações tecnológicas aos materiais com diferentes formas e dimensões submetidos ao processo [12]. As melhorias encontradas a partir do processamento e tratamentos térmicos adequados dizem respeito às propriedades mecânicas, tais como alta resistência ao escoamento mantendo razoável ductilidade além da obtenção de estrutura de grãos finos equiaxiais. Também na literatura foi verificado que a sujeição das amostras de $\mathrm{Ni}-\mathrm{Ti}$ ao processo de conformação por ECAE faz com que aumentem os contornos de grãos de alto ângulo, estrutura de sub-grãos e bandas de cisalhamento [13].

Dessa forma, os objetivos desse trabalho são fabricar ligas da família Ni-Ti com efeito de memória de forma com diferentes composições, submeter esses materiais ao processo de conformação mecânica do tipo ECAE e verificar seus efeitos nas propriedades mecânicas e térmicas.

\section{MATERIAIS E MÉTODOS}

Este trabalho foi desenvolvido no contexto de uma parceria entre a Universidade Federal de Pernambuco e a Universidade Federal de Campina Grande, através do

\footnotetext{
* Contribuição técnica ao $69^{\circ}$ Congresso Anual da ABM - Internacional e ao 14ํㅡㄹ ENEMET - Encontro Nacional de Estudantes de Engenharia Metalúrgica, de Materiais e de Minas, 21 a 25 de julho de 2014, São Paulo, SP, Brasil.
} 


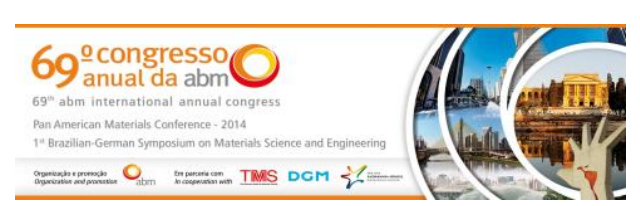

Laboratório de Materiais Inteligentes (LMI) e Laboratório Multidisciplinar de Materiais e Estruturas Ativas (LaMMEA), respectivamente. As ligas da família Ni-Ti foram selecionadas e fabricadas no LaMMEA por meio do processo Plasma Skull Push Pull (PSPP), no qual o material é fundido sobre uma fina camada dele mesmo [14]. Essa fusão foi realizada em uma máquina que utiliza o plasma térmico como meio de transmissão de energia para fundir a maioria dos metais (exceto metais com alto teor de zinco), inclusive titânio, níquel, cromo e cobalto. Para assegurar resultados satisfatórios na fusão das ligas da família Ni-Ti, utiliza-se o gás argônio no interior da câmara de fusão, que tem como característica principal ser inerte. A Figura 1 mostra a sequência do processo de fusão por plasma utilizado. Inicialmente, verificam-se os materiais dispostos no cadinho da máquina de fusão, Figura 1a. Na Figura 1b se visualiza o eletrodo rotativo de tungstênio que origina a tocha de plasma em atmosfera de argônio e provoca a fusão dos elementos puros, formando o botão mostrado na Figura 1c. Quando o metal está em seu estado fundido, este é injetado automaticamente em uma coquilha metálica, como se pode observar nas Figuras 1d e 1e, no qual é obtido o produto final da fusão, Figura $1 \mathrm{f}$.

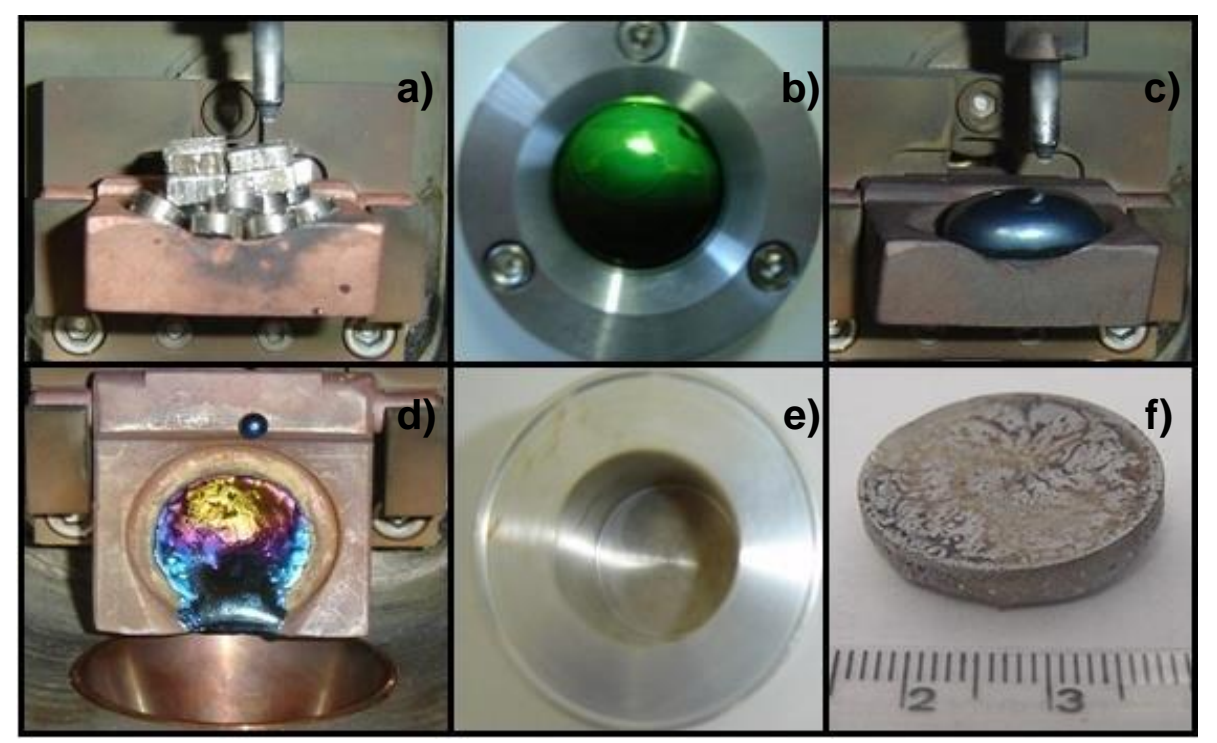

Figura 1. Sequência do processo PSPP para fusão e injeção em molde. (a) Empilhamento dos elementos da liga. (b) Tocha de plasma. (c) Botão de LMF. (d) Camada de LMF no cadinho, após a injeção. (e) Molde metálico. (f) Lingote da LMF.

As ligas fabricadas foram uma liga $\mathrm{Ni}$-Ti equiatômica, uma liga $\mathrm{Ni}$ - $\mathrm{Ti}$ rica em $\mathrm{Ni}$ e outra rica em Ti. As composições das ligas selecionadas, percentualmente em peso, foram 55Ni-Ti, 55,3Ni-Ti e 54,7Ni-Ti, respectivamente. Para assegurar a homogeneidade química dos lingotes fabricados, realizou-se um tratamento térmico de homogeneização por 2 horas num forno de mufla a $900^{\circ} \mathrm{C}$, seguido de têmpera em água a temperatura ambiente. Os lingotes foram cortados numa cortadeira de precisão com disco diamantado, visando a confecção de amostras em forma prismática para o processo de ECAE. Os prismas quadrados foram cortados com seção transversal de $5 \times 5 \mathrm{~mm}^{2}$, os quais passaram pelo processo de extrusão, realizado numa matriz confeccionada com ângulo de $120^{\circ}$ entre os canais internos. A preparação das amostras de Ni-Ti para a conformação mecânica de ECAE incluiu um tratamento térmico que consistiu no aquecimento das amostras a $850^{\circ} \mathrm{C}$, temperatura na qual permaneceram por 1 hora e posterior resfriamento em água

* Contribuição técnica ao 69 Congresso Anual da ABM - Internacional e ao 14ํㅡㄹ ENEMET - Encontro Nacional de Estudantes de Engenharia Metalúrgica, de Materiais e de Minas, 21 a 25 de julho de 2014, São Paulo, SP, Brasil. 


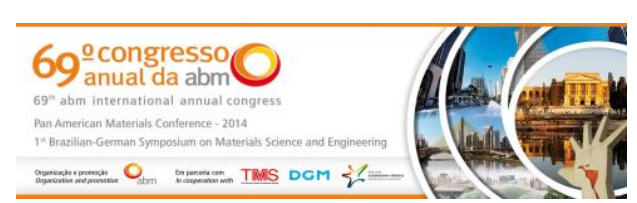

com gelo, provocando a têmpera no material. Este tratamento foi realizado para conformar as amostras em temperatura ambiente, assim como executado por Shahmir [15].

Para cada amostra do material, antes e após o processo de conformação mecânica por extrusão, foram realizados ensaios de microdureza e calorimetria diferencial exploratória (DSC). Os dados dos ensaios de microdureza foram coletados a partir de 10 indentações com distância de $1 \mathrm{~mm}$ entre elas e carga de penetração de $300 \mathrm{gf}$ por um período de $15 \mathrm{~s}$, enquanto no DSC foram realizados ciclos de aquecimento e resfriamento com taxa de $10^{\circ} \mathrm{C} / \mathrm{min}$.

\section{RESULTADOS E DISCUSSÃO}

Os materiais foram fundidos no forno de fusão à plasma, do qual foi conseguido o produto final em forma de barra prismática. As barras passaram por um processo de corte, do qual se obtiveram as lâminas para a realização da conformação plástica, como mostrado na Figura 2.
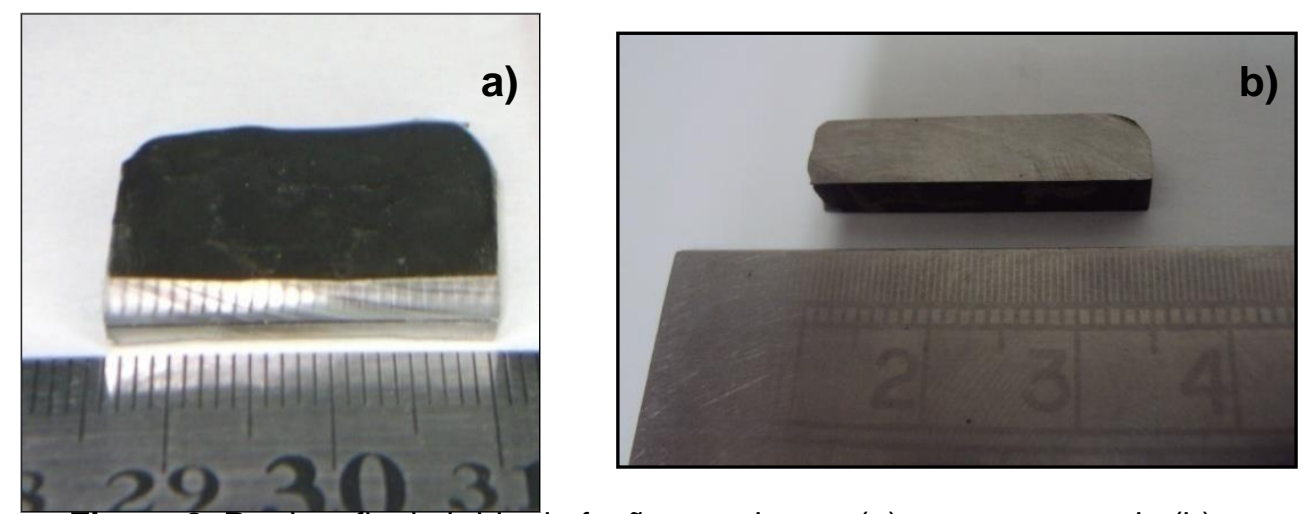

Figura 2. Produto final obtido da fusão por plasma (a) e tarugo cortado (b).

Após o corte dos tarugos prismáticos foram realizados os ensaios de DSC para as três ligas fabricadas, a saber, liga $\mathrm{Ni}$-Ti equiatômica, liga $\mathrm{Ni}$-Ti rica em $\mathrm{Ni}$ e liga $\mathrm{Ni}-\mathrm{Ti}$ rica em Ti. A Figura 3 mostra as curvas de calorimetria para as três ligas logo após sua fabricação e homogeneização.

Para todas as ligas se consegue observar a presença da transformação martensítica termoelástica reversível, conforme demonstram os picos nas curvas, tanto no aquecimento quanto no resfriamento. De forma geral, a transformação de fase nas ligas do sistema Ni-Ti ocorre em duas etapas, em conformidade com a literatura [1]. Durante o resfriamento inicia-se a transformação da austenita (B2) na fase $R$ à temperatura $R_{s}$, responsável pelo aparecimento de um pico no fluxo de calor, e posteriormente a fase $R$, com estrutura romboédrica. Continuando o resfriamento, esta fase se transforma numa estrutura martensítica monoclínica (B19'). Durante o aquecimento ocorre a transformação inversa (martensita $\rightarrow$ austenita), que é caracterizada pela inflexão na curva calorimétrica de aquecimento. A transformação como descrita anteriormente pode ser visualizada na Figura 3c, onde se consegue visualizar claramente todas as etapas da transformação. Para as outras ligas fabricadas e testadas na mesma condição, o ensaio de DSC não detectou de forma evidente a fase intermediária durante o resfriamento, a qual provavelmente está justaposta ao pico durante a transformação martensítica direta. Por meio dos resultados iniciais também se consegue verificar que a técnica de obtenção das ligas se mostra bastante eficiente, tendo em vista que a liga equiatômica apresentou

\footnotetext{
* Contribuição técnica ao $69^{\circ}$ Congresso Anual da ABM - Internacional e ao 14ํㅡㄹ ENEMET - Encontro Nacional de Estudantes de Engenharia Metalúrgica, de Materiais e de Minas, 21 a 25 de julho de 2014, São Paulo, SP, Brasil.
} 
temperaturas de transformação mais elevadas que a liga com maior percentual de níquel e mais baixas que a liga com maior teor de titânio em sua composição.
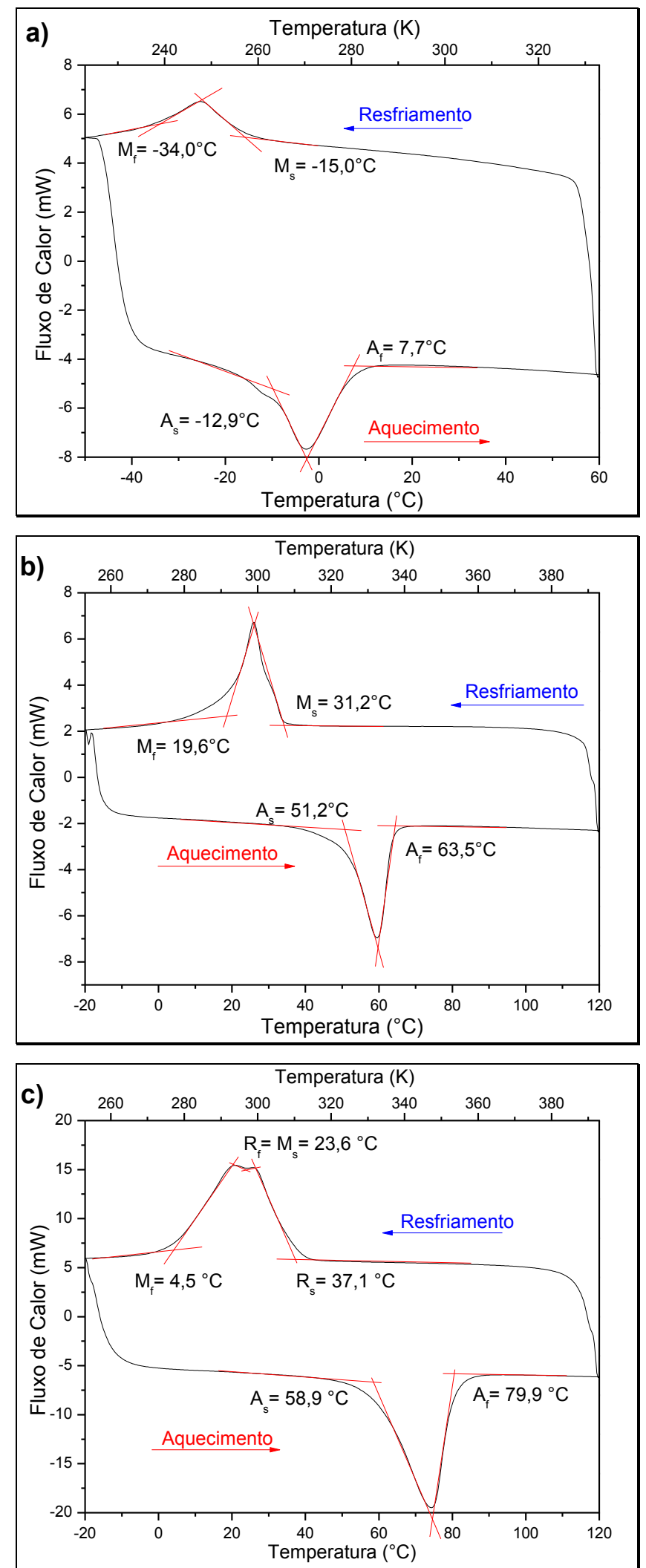

Figura 3. Curvas de calorimetria das ligas $\mathrm{Ni}$-Ti rica em $\mathrm{Ni}$ (a), equiatômica (b) e rica em $\mathrm{Ti}$ (c).

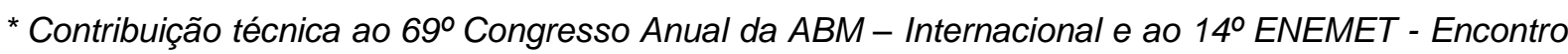
Nacional de Estudantes de Engenharia Metalúrgica, de Materiais e de Minas, 21 a 25 de julho de 2014, São Paulo, SP, Brasil. 


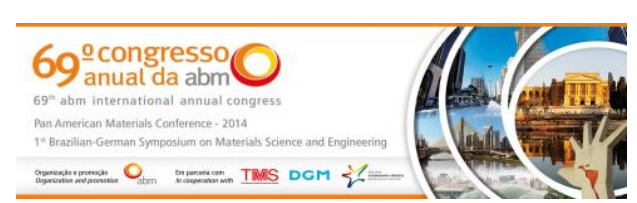

O ensaio de microdureza foi realizado para as amostras também logo após a homogeneização das ligas. Verifica-se através da Tabela 1 que a liga Rica em Ni apresenta dureza mais baixa que as outras ligas, o que se deve ao maior percentual deste elemento na liga. Além disso, os ensaios de microdureza foram conduzidos em temperatura ambiente e a única amostra ensaiada na fase austenítica foi a Rica em Ni, uma vez que as temperaturas de transformação martensítica inversa $\left(A_{s}\right.$ e $\left.A_{f}\right)$ estão abaixo da temperatura ambiente. A fase austenita possui resistência mecânica maior que a da martensita termoelástica, presente nas LMF estudadas. Este fato é a causa pela qual a liga Equiatômica apresenta resultados de microdureza pouco maiores que a da liga Rica em Ni. Possivelmente, a liga Equiatômica, durante o ensaio, já possuía um pequeno percentual da fase austenita em sua estrutura, enquanto que para a liga Rica em Ti todo o ensaio foi realizado na fase martensítica. Devido a esse fato a microdureza da liga Equiatômica apresenta valores um pouco superiores àqueles que apresentariam se estivesse totalmente na fase martensítica. Embora esteja no estado martensítico à temperatura ambiente, a liga Rica em $\mathrm{Ti}$ apresenta grau de resistência superficial superior às ligas com menor teor de $\mathrm{Ti}$ em sua composição.

Tabela 1. Dados do ensaio de microdureza para as ligas homogeneizadas

\begin{tabular}{|l|c|}
\hline \multicolumn{1}{|c|}{ Ligas Homogeneizadas } & Microdureza $(\mathrm{HV})$ \\
\hline Rica em Ni & $269.4 \pm 6.5$ \\
\hline Equiatômica & $287.7 \pm 14.2$ \\
\hline Rica em Ti & $325.3 \pm 21.9$ \\
\hline
\end{tabular}

Em seguida, realizou-se a extrusão em canal angular nas amostras, as quais foram submetidas aos ensaios de calorimetria e microdureza para efeito comparativo. A Figura 4 mostra as curvas obtidas dos ensaios de DSC para as amostras extrudadas.

Verifica-se que o grau de deformação do material foi intenso quando da passagem através dos canais da matriz, haja vista que as temperaturas de transformação das ligas foram reduzidas, se comparadas às amostras homogeneizadas. Além disso, em trabalho publicado recentemente por Silva [16], verifica-se que amostras das mesmas ligas sendo submetidas a conformação do tipo laminação demonstraram temperaturas de transformação mais elevadas, o que indica menor intensidade dos efeitos da conformação sobre as propriedades térmicas das ligas.

A amostra Rica em Ni, que anteriormente à conformação já apresentava temperaturas de transformação baixas, não exibiu as curvas características de transformação de fase durante o ciclo térmico de aquecimento e resfriamento. Isto está possivelmente relacionado à inibição da transformação martensítica, induzida pelo estado de tensões provocado pela conformação mecânica, como identificado no trabalho realizado por Figueiredo [17]. A amostra da liga Equiatômica apresentou a transformação martensítica direta ocorrendo em duas etapas. Esse fato pode ter ocorrido justamente devido à introdução de defeitos na amostra, levando as temperaturas de transformação martensítica diretas a níveis inferiores, fazendo com que houvesse uma desagregação entre as transições da fase $\mathrm{R}$ e martensítica. $\mathrm{Na}$ amostra Rica em Ti se observam as transformações, tanto direta quanto inversa, ocorrendo em uma única etapa, ou seja, austenita $\rightarrow$ martensita (direta) e martensita $\rightarrow$ austenita (inversa) sem passar por uma fase intermediária.

\footnotetext{
* Contribuição técnica ao 69ำ Congresso Anual da ABM - Internacional e ao 14ํㅡㄹ ENEMET - Encontro Nacional de Estudantes de Engenharia Metalúrgica, de Materiais e de Minas, 21 a 25 de julho de 2014, São Paulo, SP, Brasil.
} 

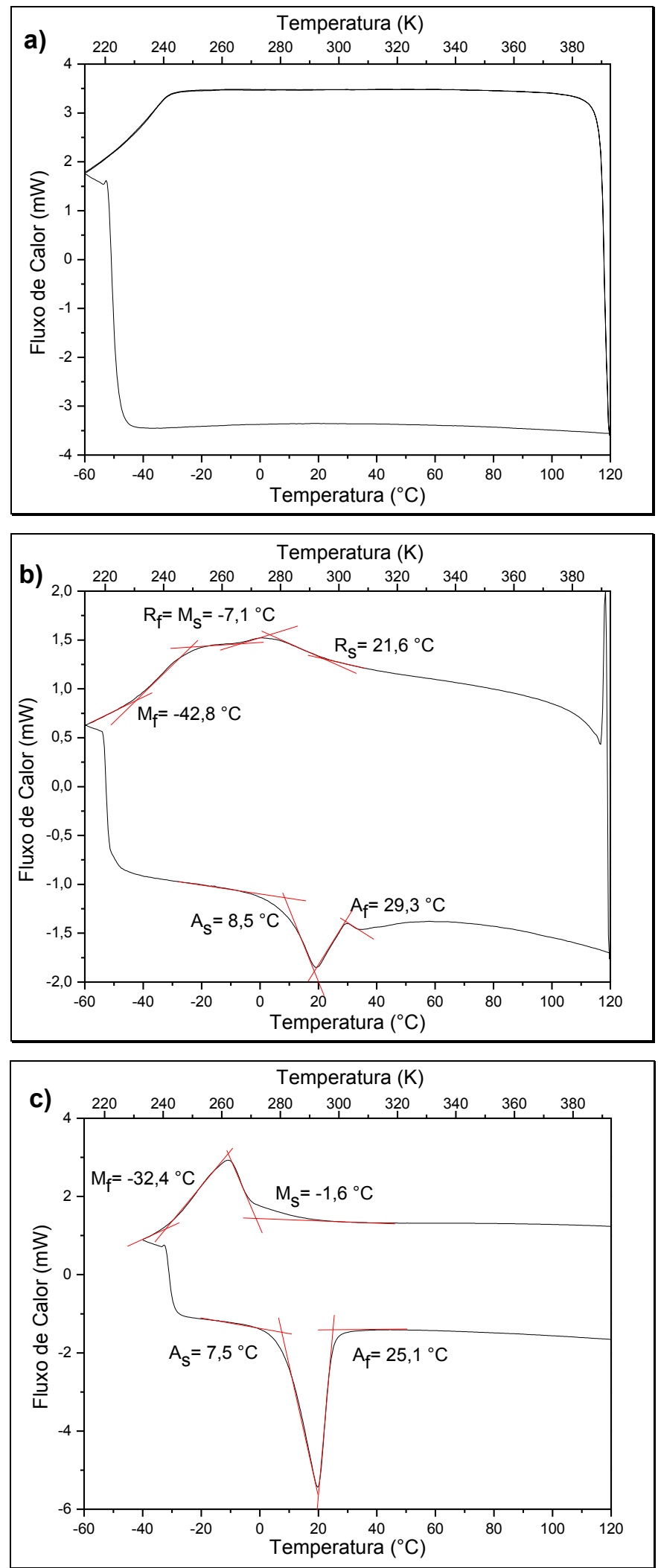

Figura 4. Curvas de calorimetria das ligas $\mathrm{Ni}-\mathrm{Ti}$ rica em $\mathrm{Ni}$ (a), equiatômica (b) e rica em $\mathrm{Ti}$ (c) extrudadas.

A Tabela 2 sintetiza os resultados das amostras que foram submetidas ao processo de ECAE, a partir da qual se percebe que todas as ligas tiveram aumento da

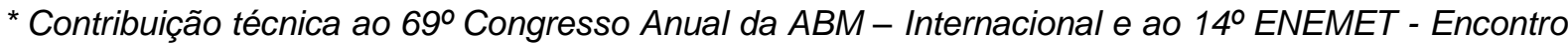
Nacional de Estudantes de Engenharia Metalúrgica, de Materiais e de Minas, 21 a 25 de julho de 2014, São Paulo, SP, Brasil. 


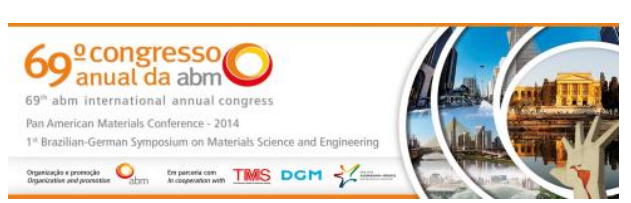

microdureza devido ao encruamento imposto pelas deformações plásticas. Inclusive, os níveis de resistência superficial encontrados para estas amostras foram mais elevados que os resultados obtidos em amostras laminadas [16], o que indica uma coerência com os resultados dos termogramas obtidos dessas ligas. Portanto, 0 grau de endurecimento nas amostras extrudadas é mais elevado que nas amostras laminadas, ou seja, a introdução de defeitos no material provocada pelo processo de conformação mecânica por extrusão se apresenta mais severo que a laminação. Dessa forma, nota-se que as deformações plásticas impostas aos materiais influenciam diretamente tanto nas propriedades mecânicas quanto nas temperaturas de transformação de fase das LMF.

Tabela 2. Dados do ensaio de microdureza para as ligas extrudadas

\begin{tabular}{|l|c|c|}
\hline \multirow{2}{*}{\multicolumn{1}{|c|}{ Amostras }} & \multicolumn{2}{c|}{ Microdureza $(\mathrm{HV})$} \\
\cline { 2 - 3 } & Homogeneizada & Após ECAE \\
\hline Rica em Ni & $269.4 \pm 6.5$ & $373,1 \pm 10,9$ \\
\hline Equiatômica & $287.7 \pm 14.2$ & $380,8 \pm 13,8$ \\
\hline Rica em Ti & $325.3 \pm 21.9$ & $398,2 \pm 14,4$ \\
\hline
\end{tabular}

\section{CONCLUSÃo}

A fabricação das ligas com efeito de memória de forma por meio do processo de fusão por plasma foi realizada com êxito, através da qual se percebeu que as temperaturas de transformação encontradas para as amostras de diferentes composições apresentaram resultados bastante coerentes, sendo mais altas para a liga com maior percentual de titânio e mais baixas para aquelas com maior percentual de níquel. A conformação mecânica do tipo extrusão demonstrou que a introdução de defeitos promoveu aumento da resistência mecânica das amostras, devido à redução do tamanho de grãos causada pelos esforços de cisalhamento da deformação plástica, além da introdução de defeitos inerentes ao processo. Trabalhos em andamento com análise microestrutural já estão sendo feitos, através doa quais se confirma a obtenção de granulação mais fina em amostras submetidas ao processo de ECAE. Concomitantemente, as temperaturas críticas de transformação de fase das ligas foram reduzidas ou até mesmo inibidas, de onde se conclui que existe maior dificuldade, ou seja, maior necessidade de energia para se completar integralmente as transformações. Dessa forma, foi verificado que, para aplicações reais de ligas da família NiTi com memória de forma em situações em que 0 material for submetido à deformação mecânica, deve-se levar em consideração o fato de que suas temperaturas de transformação sofrerão decréscimo após o processo de conformação.

\section{Agradecimentos}

Os autores agradecem ao Conselho Nacional de Desenvolvimento Científico e Tecnológico (CNPq) e a FACEPE pelo apoio aos projetos em andamento, bem como a CAPES pela concessão da bolsa de doutorado ao primeiro autor por meio do Programa de Pós-Graduação em Engenharia Mecânica da UFPE.

\footnotetext{
* Contribuição técnica ao $69^{\circ}$ Congresso Anual da ABM - Internacional e ao 14ํㅡㄹ ENEMET - Encontro Nacional de Estudantes de Engenharia Metalúrgica, de Materiais e de Minas, 21 a 25 de julho de 2014, São Paulo, SP, Brasil.
} 


\section{REFERÊNCIAS}

1 Otsuka K, Wayman CM. Shape Memory Materials, Edited by K. Otsuka and C. M. Wayman, Cambridge University Press, Cambridge, England, 1998.

2 Segal VM. Materials processing by simple shear. Materials Science and Engineering,1995; A197: 157-164.

3 Araújo CJ, Gomes AAC, Silva JA, Cavalcanti AJT, Reis RPB, Gonzalez CH. Fabrication of shape memory alloys using the plasma skull push-pull process. Journal of Materials Processing Technology, 2009; 209: 3657-3664.

4 Lagoudas DC. Shape Memory Alloys - Modeling and Engineering Applications. Springer: New York, 2008.

5 Feninat FE, Laroche G, Fiset M, Mantovani D. Shape Memory Materials for Biomedical Applications. Advanced Engineering Materials, 2002: 4: 91-104.

6 Morgan NB. Medical shape memory alloy applications - the market and its products. Materials Science and Engineering, 2004; 378: 16-23.

7 Yamauchi K, Ohkata I, Tsuchiya K, Miyazaki S. Shape Memory and Superelastic Alloys: Applications and Technologies. Woodhead Publishing: Cambridge, 2011.

8 Silva NJ, Silva MM, Gomes AAC, Araújo CJ, Santos MA., Análise prospectiva da aplicabilidade de atuadores de ligas com memória de forma no setor de petróleo e gás. CONEM 2008, p. 1- 10.

9 Petrini L, Migliavacca F. Biomedical Applications of Shape Memory Alloys. Journal of Metallurgy, 2011: 15.

10 Segal, V.M. Materials processing by simple shear. Materials Science and Engineering, 1995; A197: 157-164.

11 Kockar B, Karaman I, Kim JI, Chumlyakov Y. A method to enhance cyclic reversibility of $\mathrm{NiTiHf}$ high temperature shape memory alloys. Scripta Materialia, 2006; 54: 2203-2208.

12 Yamagushi DH, Fujinami T, Nemoto M, Langdon TG. Factors affecting grain refinement in equal-channel angular pressing. Materials Science Forum, 2000; 331-337: 607- 612.

13 Lucas FLC, Guido V, Kafer KA, Bernardi HH, Otubo J. ECAE Processed NiTi Shape Memory Alloy. Materials Research, no.ahead, pp.0-0. ISSN 1516-1439, 2014.

14 Discovery Plasma: Manual de Instalação, Operação e Manutenção. EDG Equipamentos e Controles Ltda. 2005.

15 Shahmir H, Ahmadabadi MN, Arani MM, Langdon TR., The processing of NiTi shape memory alloys by equal-channel angular pressing at room temperature. Materials Science \& Engineering, 2013; 576: 178-184.

16 Silva NJ, Gonzalez CH, Oliveira CAN, Júnior DRL, Araújo CJ. Influência da Laminação nas Propriedades de Ligas Ni-Ti com Efeito Memória de Forma. Anais do CBECiMat 2012: $1-9$.

17 Figueiredo AM, Modenesi P, Buono V. Low-cycle fatigue life of superelastic NiTi wires. International Journal of Fatigue, 2009; 31: 751 - 758.

\footnotetext{
* Contribuição técnica ao $69^{\circ}$ Congresso Anual da ABM - Internacional e ao 14ํㅡㄹ ENEMET - Encontro Nacional de Estudantes de Engenharia Metalúrgica, de Materiais e de Minas, 21 a 25 de julho de 2014, São Paulo, SP, Brasil.
} 